\title{
A genetic study of neurofibromatosis type 1 (NF1) in south-western Ontario. II A PCR based approach to molecular and prenatal diagnosis using linkage
}

Molecular Medical Genetics Program, Child Health

Research Institute, Children's Hospital of Western Ontario, 800 Commissioner's Road East, London,

Ontario, Canada N6C 2 V5.

D I Rodenhiser

P J Ainsworth

M B Coulter-Mackie

$S M$ Singh

J H Jung

\section{Department of}

Pediatrics, University of Western Ontario, London, Ontario, Canada N6C 2V5. D I Rodenhiser P J Ainsworth M B Coulter-Mackie $S M$ Singh $\mathrm{J} H$ Jung

Department of Biochemistry, University of Western Ontario, London, Ontario, Canada N6C 2 V5.

M B Coulter-Mackie

Department of Zoology, University of Western Ontario, London, Ontario Canada N6C 2 V5. $S M$ Singh

Children's Psychiatric Research Institute, London, Ontario, Canada N6C 2V5. M B Coulter-Mackie

Correspondence to Dr Rodenhiser.

Received 3 August 1992 Revised version accepted 24 October 1992.

D I Rodenhiser, P J Ainsworth, M B Coulter-Mackie, S M Singh, J H Jung

\begin{abstract}
Neurofibromatosis type 1 (NF1) is a common, autosomal dominant genetic disorder with a variety of highly variable symptoms including cutaneous manifestations (such as café au lait spots), Lisch nodules, plexiform neurofibromas, skeletal abnormalities, an increased risk for malignancy, and the development of learning disabilities. The wide clinical variability of expression of the disease phenotype and high (spontaneous) mutation rate of the NF1 gene indicate that careful clinical examination of patients and family members is necessary to provide an accurate diagnosis of the disease. Since very few NF1 mutations have been identified, and with the apparent lack of a predominant mutation in this large, highly mutable gene, molecular diagnosis of NF1 will continue to be based on haplotypes using linkage analysis. Here we report our experiences while providing a molecular diagnostic service for NF1 in the ethnically diverse region of south-western Ontario. Molecular diagnoses with at least one informative probe/enzyme combination are reported for 19 families including two families requesting prenatal diagnosis for NF1. We have augmented the classical Southern based approach to linkage analysis with the use of PCR based assays for molecular linkage. Furthermore, criteria have been established in our laboratory for executing molecular linkage based on heterozygosity values, recombination fractions, and the use of intragenic probes/markers.

(f Med Genet 1993;30:363-8)
\end{abstract}

Neurofibromatosis type 1 (NF1) is a common, autosomal dominant genetic disorder with highly variable symptoms which include a variety of cutaneous manifestations (café au lait spots, axillary freckling and dermal neurofibromas), Lisch nodules, plexiform neurofibromas, and skeletal abnormalities. ${ }^{1}$ There is an increased risk for malignancy in the form of neurofibrosarcoma, optic glioma, and certain paediatric leukaemias, ${ }^{2}$ and a proportion of affected subjects manifest learning disabilities. Since up to one half of all NF1 cases result from new mutations ${ }^{3}$ careful clinical examinations are necessary, especially with respect to the parents of affected offspring, so that the origin of the NF1 mutation (that is, familial inheritance or new mutation) can be established.

The localisation of the NF1 gene to $17 \mathrm{q} 11.2$ and the cloning and characterisation of the NF1 gene itself now permit molecular diagnosis of familial NF1.46 However, very few mutations have been identified in patients and there is an apparent lack of a predominant mutation in this large, highly mutable gene. This means that molecular diagnosis of NF1 will continue to be based on haplotypes using linkage analysis. We have previously described the population genetics, familial segregation, and molecular linkage of NF1 in families from the ethnically diverse region of south-western Ontario, Canada. ${ }^{7}$ In the present paper we report our experiences in providing a molecular diagnostic service for NF1 in this region of Ontario. This paper also describes our approach to augment classical Southern blot based linkage analysis with PCR based assays which are particularly suitable in prenatal diagnosis.

\section{Materials and methods}

SAMPLE ACQUISITION

Families evaluated in this study were referred to our laboratory from the Regional Medical Genetics Centre at the Children's Hospital of Western Ontario and the five other clinical genetics centres throughout Ontario servicing a total population of nine million. Patients were diagnosed by clinical geneticists using the criteria of the NIH Clinical Consensus Symposium. ${ }^{8}$ Criteria for blood collection and shipping instructions for samples were established with all centres before receipt of any samples. Whole venous blood was collected in EDTA coated tubes. Blood was maintained at room temperature and shipped to the laboratory by courier; amniocytes and chorionic villus samples were shipped on ice. All samples were accompanied by accurate family pedigrees. Human genomic DNA was prepared using the modified protocols of Jeanpierre ${ }^{9}$ and Miller $e t$ al. ${ }^{10}$ In all cases, 1 to $2 \mathrm{ml}$ aliquots of whole blood and aliquots of precipitated DNA (under $70 \%$ ethanol) were also banked in the laboratory for future use.

MOLECULAR LINKAGE

Molecular linkage analysis was performed either by Southern analysis or PCR based 
assays using the NF1 linked markers listed in table 1. For Southern analyses DNA was digested with the appropriate restriction enzymes (table 1), blotted to nylon membranes, and hybridised with radiolabelled probes as previously described. ${ }^{7}$

For some probes we converted molecular linkage to a PCR based assay, as previously described for the NF1 linked marker pHHH202/RsaI. ${ }^{11}$ PCR primer pairs were generated which flank the polymorphic sites associated with five additional NF1 linked probe/enzyme combinations: pEW206/MspI, pF9.8/RsaI, p1 1.3C4.2/MspI, pEW207/BglII, and pEW207/HindIII. For these PCR based analyses reaction parameters recommended by the supplier of Taq polymerase (Perkin-Elmer Cetus) were modified so that each amplification reaction $(25 \mu \mathrm{l})$ included $2.5 \mu \mathrm{l}$ of $10 \times$ PCR buffer II (Perkin-Elmer Cetus), 10\% glycerol (optional for pF9.8), 25 pmol of each primer, 0.625 units of $T a q$ polymerase, and $0.5 \mu \mathrm{g}$ genomic DNA. PCR primer sequences are shown in table 2. Magnesium chloride concentrations optimised for each primer pair include: $\quad 1.5 \mathrm{mmol} / \mathrm{l} \quad \mathrm{MgCl}_{2} \quad$ (pEW206), $2 \cdot 0 \mathrm{mmol} / 1 \mathrm{MgCl}_{2}$ (p11.3C4.2), and $2.5 \mathrm{mmol} / 1$ $\mathrm{MgCl}_{2}$ (pEW207 and pF9.8). Samples were overlaid with mineral oil before amplification.

All reaction mixtures were denatured for four minutes at $94^{\circ} \mathrm{C}$ and then subjected to 30 cycles of PCR. Amplification conditions for p11.3C4.2, pEW206, pF9.8, and pEW207

Table 1 Chromosome 17 DNA markers used for molecular prediction of NF1. Rare allele frequencies are based on founder members of each family. Heterozygosity is calculated based on equilibrium $(2 p q)$.

\begin{tabular}{lllccc}
\hline Probe & Enzyme & $\begin{array}{l}\text { Relative } \\
\text { location }\end{array}$ & $\begin{array}{c}\text { Rare allele } \\
\text { frequency }\end{array}$ & $\begin{array}{c}\mathrm{H} \\
\text { (observed) }\end{array}$ & Inf (\%) \\
\hline pHHH202 & RsaI* & Proximal & $0 \cdot 36$ & $0 \cdot 46$ & $11 / 19(58)$ \\
p11.3C4.2 & MspI* & Proximal & $0 \cdot 19$ & $0 \cdot 31$ & $3 / 13(23)$ \\
pTH1719 & BglII & Proximal & $0 \cdot 40$ & $0 \cdot 48$ & $3 / 4(75)$ \\
pEvi2BB & EcoRI & Intragenic & $0 \cdot 37$ & $0 \cdot 47$ & $3 / 6(50)$ \\
Alu repeat & TaqI & Intragenic & ND & ND & $8 / 17(47)$ \\
Exon 4 & TaqI & Intragenic & ND & ND & $1 / 3(33)$ \\
p11.2C11.7 & BamHI & Distal & $0 \cdot 47$ & $0 \cdot 49$ & $5 / 7(71)$ \\
pEW206 & MspI* & Distal & $0 \cdot 28$ & $0 \cdot 40$ & $6 / 18(33)$ \\
p2.F9.8 & BglII & Distal & $0 \cdot 33$ & $0 \cdot 44$ & $4 / 5(80)$ \\
& TaqI & Distal & $0 \cdot 25$ & $0 \cdot 38$ & $1 / 3(33)$ \\
pEW207 & RsaI* & Distal & $0 \cdot 26$ & $0 \cdot 38$ & $7 / 15(47)$ \\
& BglII* & Distal & $0 \cdot 22$ & $0 \cdot 34$ & $7 / 17(41)$ \\
& HindIII* & Distal & $0 \cdot 19$ & $0 \cdot 31$ & $5 / 17(29)$ \\
\hline
\end{tabular}

*Probe/enzyme combinations for which a PCR based assay has been developed. Inf = ratio of families in which probe was informative compared to total families tested. $\mathrm{ND}=$ not determined.

Table 2 Sequence of PCR primers used in linkage analysis of NF1.

\begin{tabular}{ll}
\hline Probe (enzyme) & Primer sequence \\
\hline pHHH202 (RsaI $)^{11}$ & Forward primer \\
& 5'-ATGAACAAGTCAAGGGACAGGCTT-3' \\
& Reverse primer \\
p11.3C4.2(MspI) & ForwTGCCAAAGGTTACAGGGCTAC-3' \\
& 5'-CACAGCTTAGTTTTCAGTGACTCC-3' \\
& Reverse primer is \\
5EW206 (MspI) & Forward primer \\
& 5'-TGCAGTGTGGTGCATCATTCAGTG-3' \\
& Reverse primer \\
p2.F9.8 (RsaI) & For'-GACAGGGCCAGCCATATTCCTGAT-3' \\
& 5'-GAACTCATGGGTTCTAAGGGAGTA-3' \\
& Reverse primer \\
pEW207 (BglII, HindIII) & Forward primer \\
Reverse primer & 5'-AGGTATCAGTCAGGACCCTCTTAG-3' \\
& 5'-CTGACACTCTGGTTTCTGTAATGTG-3'
\end{tabular}

consist of: primer annealing at $65^{\circ} \mathrm{C}$ for two minutes, elongation at $72^{\circ} \mathrm{C}$ for two minutes, and denaturation at $94^{\circ} \mathrm{C}$ for one minute 10 seconds. In all cases the final amplification cycle was followed by an eight minute extension at $72^{\circ} \mathrm{C}$. PCR primers and amplification conditions for analysis of the polyallelic $A l u$ repeat marker followed the published protocols $^{1213}$ (manuscript in preparation).

After amplification RFLPs were detected by digesting $10 \mu \mathrm{l}$ PCR product in a $15 \mu \mathrm{l}$ reaction mixture containing 5 to 10 units of the appropriate enzyme (Boehringer Mannheim Canada), $1.5 \mu$ l of the appropriate $10 \times$ buffer, and $3.0 \mu \mathrm{l}$ of water (MspI and RsaI digests were performed in PCR buffer alone). This mixture was incubated for 90 minutes at $37^{\circ} \mathrm{C}$ $\left(65^{\circ} \mathrm{C}\right.$ in the case of $\left.\mathrm{Taq} \mathrm{I}\right)$. Digestion products were mixed with loading buffer containing marker dye $(20 \%$ Ficoll $400,0 \cdot 1 \mathrm{~mol} / 1$ EDTA (pH 8), $1 \%$ SDS, $0.25 \%$ bromophenol blue, and $0.25 \%$ xylene cyanol), electrophoresed on a non-denaturing $6 \%$ polyacrylamide gel, and then visualised with ethidium bromide. The sizes of PCR products and DNA fragments generated by restriction digestion are given in table 3. Analysis of linkage data generated by Southern and PCR based assays for estimation of genetic risk used the program MLINK with published recombination frequencies for $\mathrm{NF} 1$ markers. ${ }^{714}$

\section{Results}

FAMILIES

A total of 60 families was referred to the laboratory from the six Regional Medical Genetics Centres of the province of Ontario, 34 of which represent familial cases of NF1. Molecular diagnosis with at least one informative probe/enzyme combination (with a certainty of at least $95 \%$ ) has been completed for 19 families. Analyses for four families are still in progress since DNA has yet to be received from family members critical for establishing linkage. Eleven files which have been designated inactive represent families who did not wish molecular diagnosis for NF1 or where contact with the families has been lost.

Twenty-four subjects represent apparent sporadic cases of NF1. The higher than expected ratio of familial to sporadic cases of NF1 evaluated by our laboratory (34:24) reflects a selection bias earlier in our research programme when familial cases (with at least two affected subjects) were actively sought to establish linkage relationships between the DNA markers and NF1. New sporadic cases

Table 3 Size of PCR product and fragments detected by polymorphic restriction enzymes.

\begin{tabular}{llcc}
\hline Probe & Enzyme & $\begin{array}{c}\text { PCR product } \\
\text { size (bp) }\end{array}$ & $\begin{array}{c}\text { Fragment sizes } \\
\text { (bp) }\end{array}$ \\
\hline pHHH202 & RsaI & 306 & $188 / 108$ \\
p11.3C4.2 & MspI & 1080 & $920 / 160$ \\
pEW206 & MspI & 349 & $180 / 169$ \\
p2.F9.8* & RsaI & $\sim 650$ & $460 / 400,60$ \\
pEW207 & BglII & 587 & $368 / 219$ \\
& HindIII & 587 & $390 / 197$ \\
\hline
\end{tabular}

*Two constant bands are also detected $(120,80 \mathrm{bp})$. 
are now counselled and asked to participate in the continuing research which seeks to identify patient specific NF1 mutations and correlate these mutations with clinical features of NF1. To date, two such patient specific NF1 mutations have been identified by our group in a survey of 50 patients. ${ }^{15}$ For two other families referred to the laboratory, the origin of the mutation has yet to be determined owing to incomplete clinical data on particular family members.

\section{MOLECULAR LINKAGE}

Molecular linkage analysis of NF1 for 25 families involved the use of a battery of NF1 linked DNA markers flanking the NF1 locus as well as the intragenic marker pEvi2BB/EcoRI ${ }^{1616 a}$ (table 1). Observed heterozygosity and allele frequency data for many of the markers are not significantly different from published data. ${ }^{7}$ No recombinants were observed involving $\mathrm{NF} 1$ and any markers except F9.8/RsaI, where three recombinants were found in 15 meioses. For some analyses we also used two other intragenic markers, either the TaqI RFLP detected with a cDNA corresponding to NF1 exon $4^{417}$ or the polyallelic tandem repeat associated with an intragenic NF1 Alu sequence ${ }^{1213}$ (unpublished results). At least one probe/enzyme combination was found to be informative for 19 families; six families require further DNA samples to establish linkage. Fig 1A presents a pedigree and haplotype for one of our NF1 families. Several markers were informative which confirmed the clinical diagnosis of NF1 within this family. Also, a double crossover distal to the NF1 locus is carried by one affected family member. Repeat testing by Southern blotting and PCR based assays for several of the markers confirmed this recombination event. Such double crossover events are expected to be rare in the population (less than $0.5 \%$ ).

\section{PCR BASED ASSAYS}

PCR primers were developed which flank the polymorphic restriction sites detected by the following NF1 linked DNA probes: pHHH202/RsaI ${ }^{11} \quad$ pEW206/MspI, pF9.8/ RsaI, p11.3C4.2/MspI, pEW207/BglII, and pEW207/HindIII. ${ }^{14}$ Table 3 lists the size of PCR products and the DNA fragment sizes following digestion with the restriction enzyme which shows the polymorphism. For example, fig 2 shows the results generated by amplification with pEW207 primers and the HindIII and BglII site genotypes resulting from restriction digestion with each of these enzymes. Such PCR results confirmed the molecular status of all subjects previously established by Southern blot analysis (data not shown). Table 3 lists the six probe/enzyme combinations converted to PCR, the sizes of PCR product, and the DNA fragment sizes following digestion with the appropriate restriction enzyme. In all cases the results of all initial PCR based assays on a panel of control subjects were confirmed by Southern blot analysis.

\section{CRITERIA FOR USING MARKERS FOR LINKAGE} ANALYSIS

Using the genetic values for the NF1 linked DNA markers presented in table 1 and our experience in offering molecular diagnosis, a set of criteria has been established which prioritises the DNA markers used for linkage analysis in our laboratory. These criteria attempt to balance the necessity for rapid results (which may be required in the case of prenatal diagnosis), heterozygosity of a marker, and the recombination frequency between a particular marker and NF1. Our prioritised list includes (1) the use of PCR based NF1 linked markers (particularly pHHH202), (2) the use of intragenic polyallelic tandem repeat associated with an intragenic NF1 Alu sequence, ${ }^{1213}$ (3) the use of Southern based intragenic markers $\mathrm{pEvi2} \mathrm{BB}^{16}{ }^{16 a}$ and $\mathrm{TaqI} /$ exon $4,{ }^{17}$ and (4) the use of Southern blot analyses with flanking markers (particularly pTH1719 and p11.2C11.7 ${ }^{14}$ ).

\section{PRENATAL DIAGNOSIS}

Two families requested and received prenatal diagnosis for NF1. In one family the mildly affected mother (presenting with multiple café au lait spots and dermal neurofibromas) had previously given birth to a child with severe NF1 symptoms early in infancy. Prenatal diagnosis using CVS in the subsequent pregnancy indicated a low risk for a fetus carrying the NF1 mutation $(p=0.997)$. The parents elected to continue the pregnancy and an apparently unaffected child was born. The second request for prenatal diagnosis came from a couple in which the affected parent had fathered an affected child in a previous marriage (see pedigree and haplotype analysis, fig 1B). Prenatal diagnosis using CVS has been performed in this centre on two subsequent pregnancies resulting from the second marriage. The first prenatal diagnosis indicated a low risk for a fetus carrying the NF1 mutation of paternal origin $(p=0.999)$. An apparently unaffected male child was born. Prenatal diagnosis for the second pregnancy indicated a high risk for a fetus carrying the NF1 mutation $(p=0.997)$.

\section{Discussion}

In this paper we report our experiences while providing a molecular diagnostic service for NF1 in this region of Ontario. Of particular interest, we have augmented the classical Southern blot based linkage analysis with PCR based assays which are particularly suitable in prenatal diagnosis. In conjunction with our PCR based assay for pHHH202, ${ }^{11}$ six additional NF1 linked probe-enzyme combinations have been converted to PCR. PCR based methods are advantageous in that they can replace or augment the time consuming and more cumbersome Southern analysis with a sensitive, rapid, and non-radioactive approach to linkage analysis. These methods are especially desirable in prenatal diagnosis where gestational age of the fetus is an important 
A

Fam:15NF
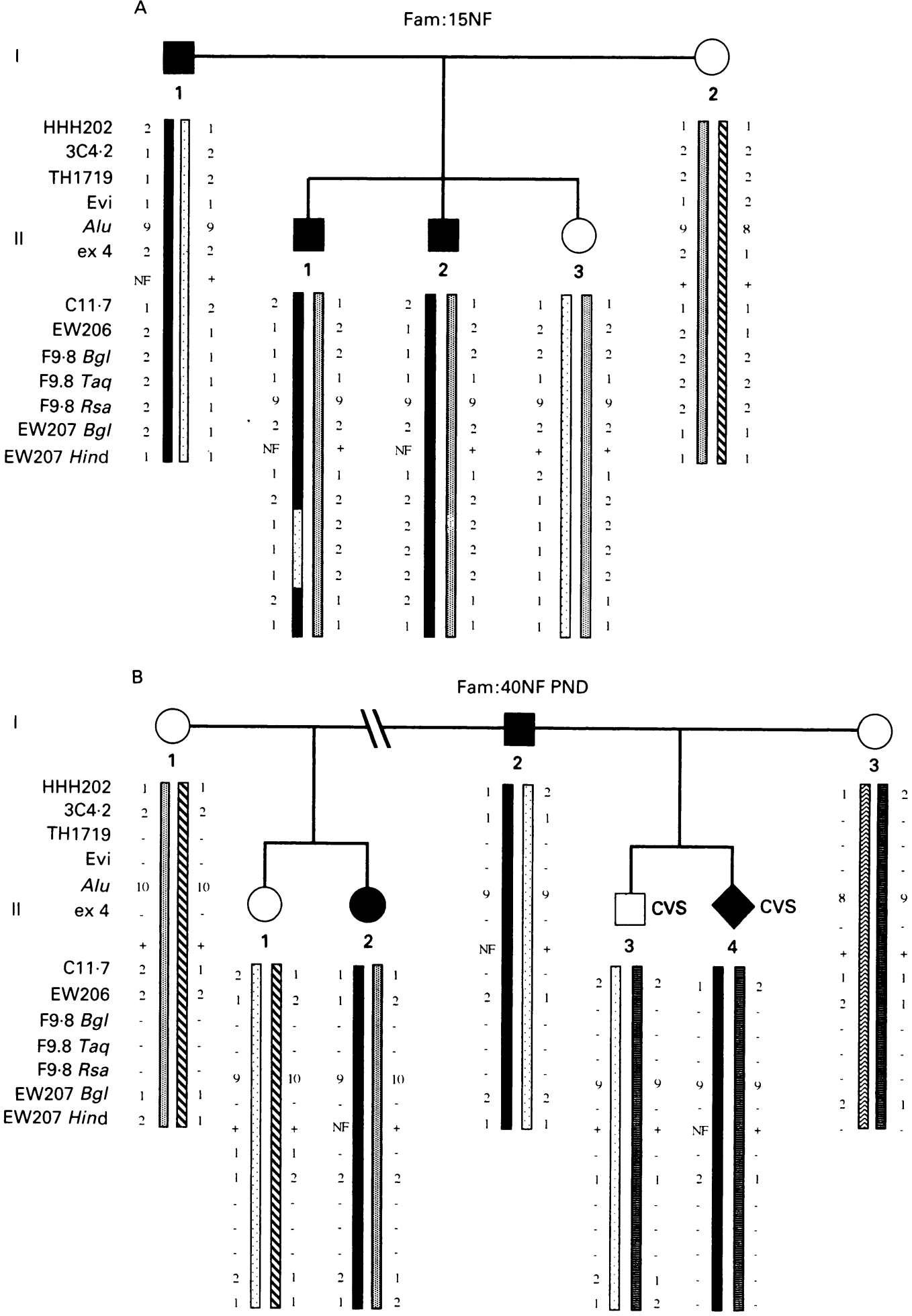

Figure 1 Application of haplotype analysis of NF1 markers in two families: ( $A$ ) molecular diagnosis and ( $B$ ) prenatal diagnosis. Sequence of the probe/enzyme combinations used follow table 1. Data which are unavailable are designated - . Localisation and segregation of the NF1 locus is designated where NF= mutation and $+=$ normal. The chromosome carrying the affected haplotype in each pedigree is coloured black.

consideration. Furthermore, this procedure can be adapted to silver stain protocols using the Phastgel system (Pharmacia) thus eliminating the need to handle potentially toxic acrylamide monomer and ethidium bromide.

Our criteria for use of available markers for linkage analysis attempt to balance the need for rapid results and the expense of the test (with respect to labour and resources) with the effectiveness of the particular markers being used (that is, heterozygosity and recombination fre- quency). As a result, we routinely use PCR based assays in the first stage of our analyses, then use the polyallelic $A l u$ repeat assay, followed by Southern based analyses using intragenic and linked markers (if appropriate). While PCR based assays provide a sensitive and rapid approach to linkage analysis, great care must be taken to ensure accurate results. In order to accomplish this, we assign all workers separate PCR kits with aliquoted reagents and PCR reaction mixtures are pre- 


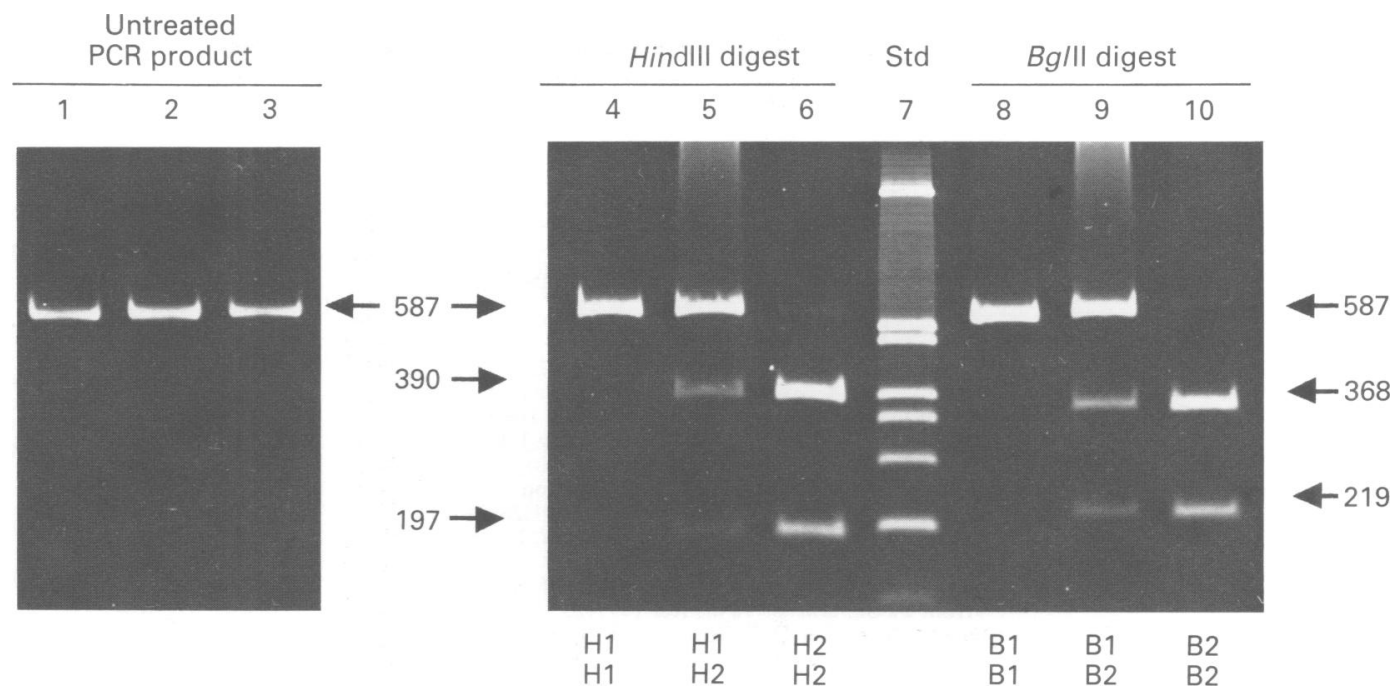

Figure 2 PCR amplification of genomic DNA to reveal the HindIII and BglII RFLP sites detectable by pEW207. The 587 bp PCR product from genomic DNA of three subjects (lanes 1, 2, 3) was digested with HindIII (lanes 4, 5 , 6 ) or BglII (lanes 8, 9, 10). HindIII digests show the H1 allele lacking the polymorphic HindIII restriction site and the $\mathrm{H} 2$ allele containing the polymorphic HindIII restriction site yielding 197 and 390 by HindIII fragments (lane 5: H2H1 heterozygote; lane 6: H2 homozygote). BglII digests show the B1 allele lacking the polymorphic $B g l I I$ restriction site and the B2 allele containing the polymorphic BglII restriction site yielding 219 and 368 bp BglII fragments (lane 9: B1B2 heterozygote; lane 10: B2 homozygote). Lane 7 shows the pBR322/HinfI molecular markers.

pared in a 'clean' room with dedicated micropipettors and equipment. No PCR amplified samples are permitted in this work area, and all possible steps are taken in order to eliminate the potential for cross contamination of PCR reaction mixtures. All amplification runs include negative controls (lacking DNA template) and positive controls (containing DNA template from a known heterozygote for a particular probe/enzyme combination). All amplifications and restriction digests are performed in duplicate and in separate runs. Furthermore, to rule out incomplete digestion of PCR product by restriction enzymes, another PCR product (possessing the appropriate restriction site) may be added to the reaction so that complete digestion can be monitored by cleavage of the internal control.

Prenatal diagnosis using CVS tissue requires special consideration in the light of possible maternal contamination. In conjunction with the precautions listed above, we routinely use PCR primers complementary to sequences flanking VNTR loci $^{18}$ to rule out maternal contamination of CVS samples. In addition fetal sex is determined using the amelogenin locus, ${ }^{19}$ although this information is not routinely included in the clinical report.

Several groups have reported their experiences with presymptomatic detection and prenatal testing of NF1.1420 DNA testing for NF1 by linkage analysis may be most useful as an adjunct to the clinical diagnosis of familial NF1 in young children, for prenatal testing, and when resources available for a complete clinical examination are limited. ${ }^{21}$ In our clinical setting, we have neither formally documented the reasons patients have requested molecular diagnosis nor assessed parental attitudes in our population toward molecular diagnosis and prenatal testing for NF1. However, molecular testing has been used to confirm clinical diagnoses and to ascertain molecular status to allow future reproductive choice.
Two families have requested and received prenatal testing for NF1 and in several cases molecular diagnosis has been requested by families with young children who have yet to exhibit any manifestations of the disease. Formal evaluation of the parental perceptions of molecular testing would be valuable in assessing the impact of molecular diagnosis of genetic diseases such as NF1 on the effective and efficient delivery of limited health care resources.

Attempts by a number of laboratories to characterise family specific mutations within the NF1 gene or to identify common mutations have met with only limited success. ${ }^{415}$ Therefore, it is apparent that for the immediate future molecular diagnosis of NF1 will continue to depend on the results of linkage analysis, as more NF1 families request information concerning their genetic status. The augmentation of classical linkage analyses with PCR based assays has, in our experience, facilitated molecular diagnosis of this most common autosomal dominant disease characterised by extensive clinical heterogeneity.

We thank Drs R L White and F S Collins for providing the linked markers used in this study. We are indebted to our NF1 patients and their families for their cooperation. We acknowledge the technical support of J Elliott and A Stuart, the efforts of $\mathrm{N}$ Scanlan, G Sheridan, J Kane, Drs V M Siu, and H C Soltan in the Regional Medical Genetics Centre at the Children's Hospital of Western Ontario and the geneticists and genetic counsellors at the five other genetics centres throughout Ontario. We also wish to thank the Foundation of the Children's Hospital of Western Ontario for their continuing funding of NF1 research within the Child Health Research Institute.

1 Riccardi VM. Type 1 neurofibromatosis and the pediatric patient. Curr Probl Pediatr 1992;22:66-107.

2 Rubenstein AE, Korf BR. Neurofibromatosis: a handbook for 
patients, families and healthcare professionals. New York: Thieme, 1990.

3 Huson SM, Compston DAS, Clark P, Harper PS. A genetic study of von Recklinghausen neurofibromatosis in south east Wales. I. Prevalence, fitness, mutation rate, and the effect of parental transmission on severity. $\mathcal{f}$ Med Genet 1989;26:704-11.

4 Cawthon RW, Weiss R, Xu G, et al. A major segment of the neurofibromatosis type 1 gene: cDNA sequence, genomic neurofibromatosis type 1 gene: cDNA sequence, genomic

5 Marchuk DA, Saulino AM, Tavakkol R, et al. cDNA cloning of the type 1 neurofibromatosis gene: complete sequence of the NF1 gene product. Genomics

6 Wallace MR, Marchuk DA, Andersen LB, et al. Type disrupted in three NF1 patients. Science 1990;249:181-6.

7 Rodenhiser DI, Coulter-Mackie MB, Jung JH, Singh SM. A genetic study of neurofibromatosis 1 in south-western Ontario. I Population, familial segregation of phenotype, and molecular linkage. $\mathcal{F}$ Med Genet 1991;28:746-51.

8 Stumpf DA, Alksne JF, Annegers JF, et al. Neurofibromatosis. Arch Neurol 1988;45:575-8.

9 Jeanpierre M. A rapid method for purification of DNA from blood. Nucleic Acids Res 1977;15:9611-12.

10 Miller SA, Dykes DD, Polesky HF. A simple salting out method for extracting DNA from human nucleated cells. Nucleic Acids Res 1988;16:1213.

11 Ainsworth PJ, Rodenhiser DI. Rapid nonradioactive detection by PCR of HHH202/Rsa I linked to neurofibromatosis type I. Am f Hum Genet 1991;49:1098-9.

12 Xu G, Nelson L, O'Connell P, White R. An Alu polymorphism intragenic to the neurofibromatosis type 1 gen (NF1). Nucleic Acids Res 1991;19:3764.

13 Lazaro C, Ravella A, Casals T, Volpini V, Estivill X.
Prenatal diagnosis of sporadic neurofibromatosis 1. Lancet 1992;339:119-20.

14 Ward K, O'Connell P, Carey JC, et al. Diagnosis of neurofibromatosis I by using tightly linked, flanking DNA markers. Am 7 Hum Genet 1990;46:943-9.

15 Ainsworth PJ, Rodenhiser DI, Costa TM. Identification and characterization of sporadic and inherited mutations in exon 4 of the neurofibromatosis (NF1) gene. Hum Genet (in press)

16 Andersen LB, Wallace MR, Marchuk DA, et al. A polymorphic cDNA probe on chromosome 17q11.2 located within the NF1 gene (D17S376). Nucleic Acids Res 1991;19:197.

$16^{\text {a }}$ Cawthon RM, Andersen LB, Buchberg AM, et al. cDNA sequence and genomic structure of EVI2B, a gene lying within an intron of the neurofibromatosis type 1 gene. Genomics 1991;9:446-60.

$17 \mathrm{Xu}$ W, Liu L, Ponder M, Ponder BAJ. A Taq polymorphism in the human NF1 gene. Nucleic Acids Res 1991;19:4570.

18 Budowle B, Chakraborty R, Giusti AM, Eisenberg AJ, Allen RC. Analysis of the VNTR locus D1S80 by the PCR followed by high resolution PAGE. Am f Hum Genet 1991;48:137-44

19 Nakahori Y, Hamano K, Iwaya M, Nakagome Y. Sex identification by polymerase chain reaction using $\mathrm{X}-\mathrm{Y}$ homologous primer. Am f Med Genet 1991;39:472-3

20 Upadhyaya M, Fryer A, MacMillan J, Broadhead W, Huson SM, Harper PS. Prenatal diagnosis and presymptomatic detection of neurofibromatosis type $1.7 \mathrm{Med}$ tomatic detection of

21 Hofman KJ, Boehm CD. Familial neurofibromatosis type 1 clinical experience with DNA testing. $f$ Pediatr 1992;120:394-8. 